\title{
Practical Remote Microscopy Using KVM Over IP
}

\author{
H.O. Colijn*, D.E. Huber**, P.C. Collins***, H.L. Fraser** \\ * Campus Electron Optics Facility, The Ohio State University, 116 W. $19^{\text {th }}$ Ave., Columbus, OH \\ 43210. \\ ** Center for the Accelerated Maturation of Materials, Materials Science \& Engineering, The Ohio \\ State University, 2041 College Rd. Columbus, OH 43210. \\ *** Quad Cities Manufacturing Laboratory, Joint Manufacturing and Technology Center at the Rock \\ Island Arsenal, Rock Island, IL 61299
}

Remote microscopy has become an important technique to leverage expensive instrumentation and provide greater access to researchers who can't be present at the instrument. There are several ways to interact with remote users: 1.) Allow "over the shoulder" imaging where the user can see the images, but not control the instrument; 2.) Allow full or limited control of the instrument by the remote user.

Currently there are expensive proprietary hardware and software remote microscopy solutions for the uni- or limited-bidirectional cases. Non-proprietary methods include software solutions such as VNC or MS Remote Desktop. In many cases, these work but allow only limited bi-directional performance such as remote diagnostics or for checking the status of an instrument but are not very useful for actual remote operation or live viewing. These software solutions also present network security issues since the instrument PC generally needs to be connected directly to the internet.

Hardware methods for remote access can provide greatly improved performance. KVM (Keyboard, Video, Mouse) switches have been in use for many years and now "KVM over IP" devices are common in the IT community for remote administration. The KVM device is inserted between the PC and the monitor, keyboard, and mouse, so anything which appears on the computer monitor can be seen remotely. Since the device is independent of the instrument PC, it uses no local computing power. Remote troubleshooting capabilities are enhanced since the host PC can even be rebooted without losing the connection.

The "SimpleLink" KVM over IP [1] devices provide hardware data encoding and a proprietary client that offers data encryption and user authentication for security. The client program can be easily configured for easy administration of multiple systems. Similar devices are available from other manufacturers. We have had very good success in operating our equipment from other locations within the department and university. The SimpleLink even has adequate performance for live focusing and stigmation, etc. over a residential cable modem connection. The operation is far better than using the software only methods. Experiments suggest, however, that for an experience that is identical to sitting at the instrument, you need $>30 \mathrm{Mbps}$ continuous data transfer rate. [2] The higher-end GlobalLink [1] device provides hardware data encoding and decoding giving an "at the instrument" feel even over a 30Mbps bandwidth connection. While the GlobaLink provided the best experience for the most demanding applications, the Simplelink provides a good balance of performance, cost, security, and ease of use.

If the instrument can be operated with only a mouse and keyboard, the KVM over IP devices work 
very well. Some instruments, however, require additional control interfaces such as TEM control pads. These specialized controls can be run remotely using standard USB over IP devices.

References

[1] www.ThinkLogical.com

[2] P. Calyam, N. Howes, A. Kalash, M. Haffner, "User and Network Interplay in Internet Telemicroscopy", ICST/ACM Immersive Telecommunications Conference (IMMERSCOM), 2007 [3] Support from the Ohio Board of Regents is gratefully acknowledged.

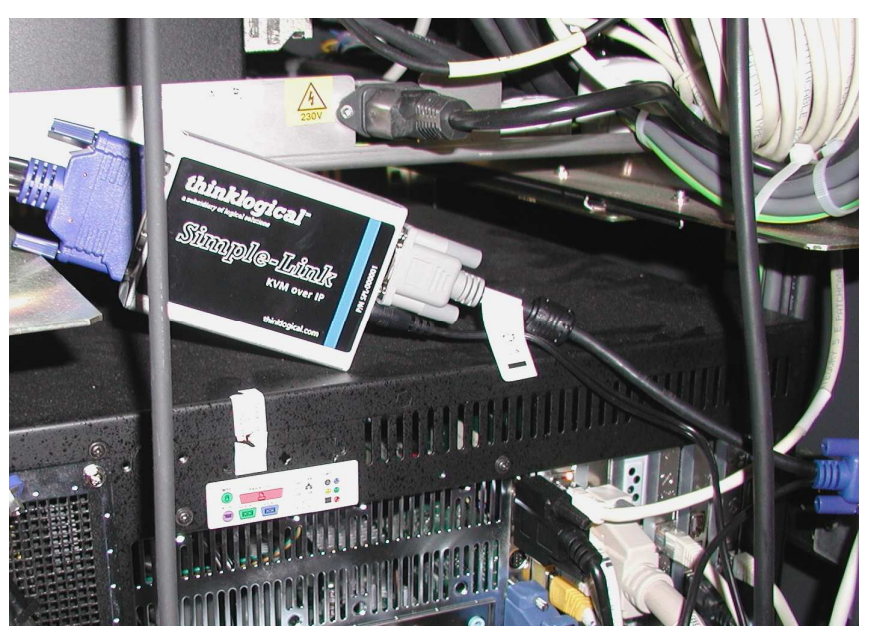

Figure 1. SimpleLink device installed on host PC

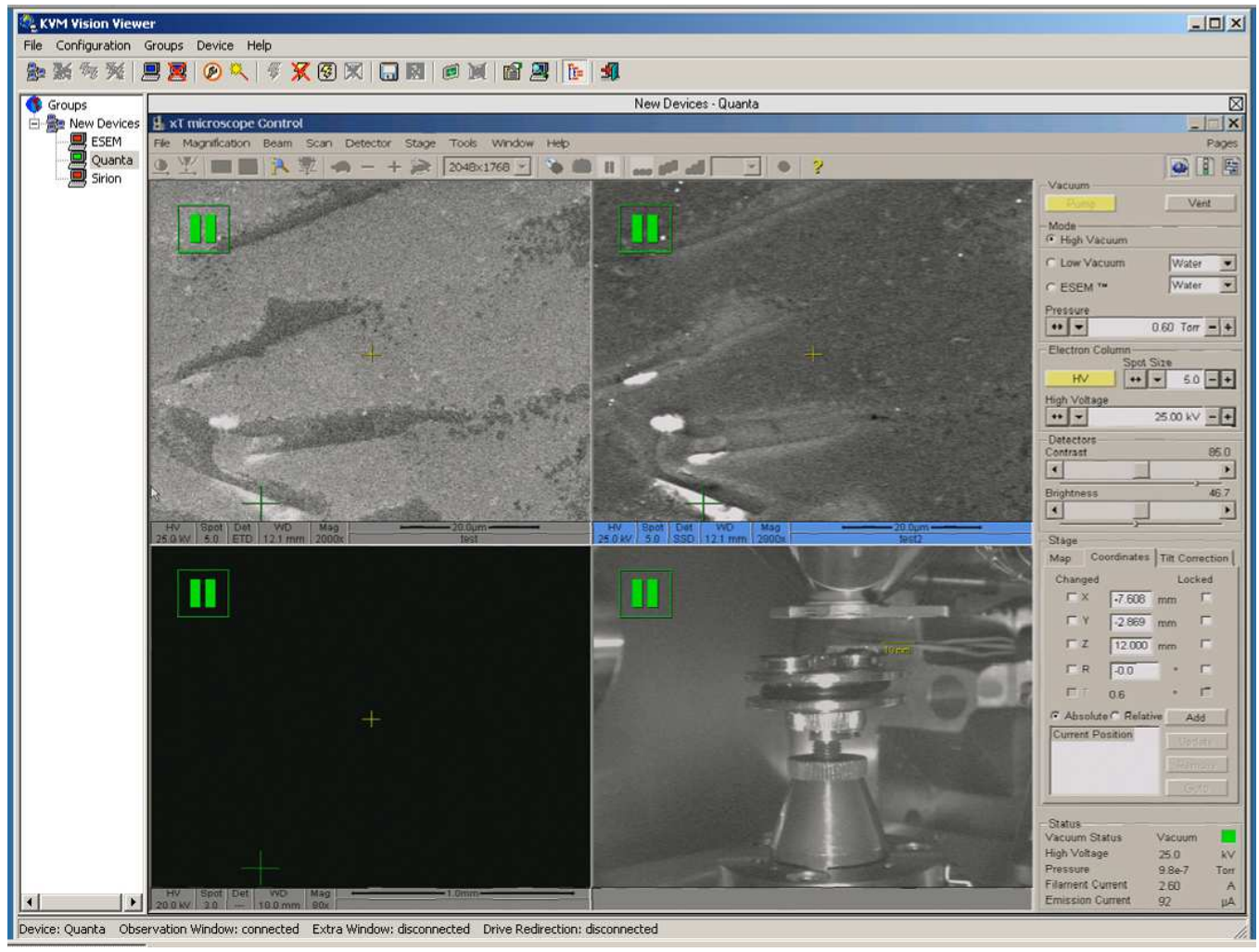

Figure 2. Screen image using the SimpleLink remote viewing program. 